\title{
Simulating Spiking Neural Networks with Timed Dataflow Graphs
}

\author{
Pan, Lei
}

IEEE

2020

Pan , L , Christophe , F , Mikkonen , T , Li , Z \& Bhattacharyya , S 2020 , Simulating Spiking Neural Networks with Timed Dataflow Graphs . in 2020 2nd IEEE International Conference on Artificial Intelligence Circuits and Systems . IEEE , IEEE International Conference on Artificial Intelligence Circuits and Systems , Genoa , Italy , 31/08/2020 . https://doi.org/10.1109/AICAS48895.2020.9C

http://hdl.handle.net/10138/322902

https://doi.org/10.1109/AICAS48895.2020.9073928

other

Downloaded from Helda, University of Helsinki institutional repository.

This is an electronic reprint of the original article.

This reprint may differ from the original in pagination and typographic detail.

Please cite the original version. 


\title{
Simulating Spiking Neural Networks with Timed Dataflow Graphs
}

\author{
Lei $\mathrm{Pan}^{1}$, Francois Christophe ${ }^{2}$, Tommi Mikkonen ${ }^{3}, \mathrm{Zhu} \mathrm{Li}^{4}$, and Shuvra S. Bhattacharyya ${ }^{1}$ \\ ${ }^{1}$ Dept. of ECE and UMIACS, University of Maryland, College Park, Maryland, USA \\ ${ }^{3}$ Dept. of Computer Science, University of Helsinki, Helsinki, Finland \\ ${ }^{4}$ Dept. of Computer Science and Electrical Engineering, University of Missouri, Kansas City, Missouri, USA
}

\begin{abstract}
This article presents a novel approach for simulating Spiking Neural Networks (SNNs) that is based on timed dataflow graphs. Whereas conventional SNN simulators compute changes in spiking neuron variables at each time step, the proposed simulation approach focuses on evaluating spike timings. This focus on evaluating when a dataflow actor (spiking neuron) reaches a new spike contributes to making spike evaluation an event-driven computation. The resulting event-driven simulation approach avoids unnecessary computations at time steps that lie between spiking events. This optimization is achieved while avoiding the large overheads associated with lookup tables that are incurred in existing event-driven approaches. Our results show identical spiking behavior compared to simulation using a conventional (time-based) simulator while providing significant improvement in execution time.
\end{abstract}

Index Terms-Dataflow, model-based design, simulation, spiking neural networks.

\section{INTRODUCTION}

The simulation of biological computation with Spiking Neural Networks (SNNs) has recently gotten attention for saving energy during computation [1], [2]. SNNs are often used in computational neuroscience for modeling biological behavior comparable to in-vitro or in-vivo experiments. In recent years, SNNs have also been applied to circuits and systems for machine learning.

In this paper, we propose a novel event-driven approach for simulating SNNs, and we present a prototype simulator called LDSS (Lightweight Dataflow SNN Simulator), which demonstrates this approach. LDSS is developed using the Lightweight Dataflow Environment (LIDE) [3], which is a tool for dataflow-based design of signal processing systems.

The simulation approach used in LDSS involves a finiteelement evaluation to predict the next time a given neuron fires a spike. This type of prediction is used to make the computation of spikings in the network event-driven instead of time-driven. Such event-driven computation is enabled by the design of SNNs as dataflow graphs, where each actor (vertex) of a graph represents a spiking neuron and encapsulates computation that predicts the next firing time of the neuron given its current state. In addition to improving simulation speed through event-driven simulation, the dataflow-based formulation of the proposed simulation approach facilitates retargetability across diverse hardware platforms, such as CPU, FPGA, or GPU platforms, or their hybrid combinations.

\section{RELATED WORK}

Various SNN simulators exist (e.g., see NEURON [4], NEST [5], Brian [6], [7]). Each simulator has its own specificity and purpose, but generally, they have evolved into providing an abstraction layer for computational neuroscientists to focus mainly on the specifications of their simulations, may it be at the molecular level or for the description of a large population of highly interconnected neurons.

SNN simulators are developed following two different approaches: time-driven or event-driven simulation, with timedriven approaches being more common. The preference for time-driven approaches stems in part from the slower speed of existing event-driven SNN simulators. This slow speed results from extensive use in existing event-driven simulators of precompiled lookup tables [8], where the dimensionality of the tables and the frequency of their access become bottlenecks. As a result, time-driven approaches outperform conventional event-driven approaches for complex networks [8]. The main contribution of this paper is to introduce a new approach to event-driven SNN simulation that is based on dataflow modeling, and event-prediction functions that are encapsulated within dataflow actors. The proposed approach is shown to outperform time-driven simulation, with the performance gap increasing as the size of the network increases. Rather than seeking to introduce a new tool in the large space of existing SNN tools, our primary contribution is to introduce new insight into the trade-off between event- and time-driven simulation, which may in turn contribute to the improvement of existing tools or development of new ones.

There are only few prior developments to our best knowledge that focus on designing and simulating SNNs with dataflow techniques. The NeuroFlow framework, which uses a proprietary dataflow engine as the hardware platform, is a noticeable example following this direction [9]. In contrast to NeuroFlow, our work applies dataflow as a programming model while imposing no constraints on the hardware platform. This facilitates retargetability to diverse platforms (e.g., single-core, multicore, or FPGA platforms), as described in Section I. Moreover, the approach we propose in this paper is entirely based on event-driven computation, whereas the NeuroFlow design computes internal parameter variations of each neuron of the network using a time-driven approach. 


\section{BACKGROUND}

\section{A. Spiking Neuron Model}

For the development of LDSS, we apply Izhikevich's simple model of spiking neurons [10] to simulate neurons in networks that are represented in LDSS. Izhikevich's model is selected as a first model for incorporation into LDSS because the model combines biological realism with computational simplicity. However, the main ideas of LDSS can be applied to any model of choice, such as AdEx [11] or Leaky Integrate-and-Fire.

A synaptic connection is often modeled as a weight $w_{i j}$ randomly generated between two neurons $i$ and $j$ with the connection going from neuron $i$ (pre-synaptic) to neuron $j$ (post-synaptic). When the pre-synaptic neuron is excitatory, the weight $w_{i j}$ is normalized to the range $[0,0.5]$. Similarly, if the pre-synaptic neuron is inhibitory, $w_{i j} \in[-1,0]$.

A network composed of such random constant synaptic weights for all its synaptic connections defines the class of pulse-coupled neural networks (PCNNs). In PCNNs, synaptic connections are fixed at the initialization of the network and weights remain constant throughout the simulation. The simulation experiments presented in this paper are based on this type of synaptic connectivity in order to present the validity of our dataflow-based and event-driven simulation approach with comparison to a reference sequential, time-driven C-language version.

Other models of synaptic connections exhibiting shortterm [12] and long-term plasticity [13] exist in the literature. These are not within the scope of this paper and will be subjects of future work in the development of LDSS.

\section{B. Dataflow Modeling}

Dataflow-based design models of signal and information processing systems are directed graph models in which graph vertices, called actors, represent computational modules, and each edge represents the communication of data from the output of one module to the input of another. A recent study illustrating the utility of dataflow techniques in the context of AI circuits and systems is presented by Li et al. [14]. For detailed background on dataflow-based modeling of signal and information processing systems, we refer the reader to [15], [16].

For implementation of dataflow graphs in LDSS, we employ the Lightweight Dataflow Environment (LIDE) [3]. LIDE includes application programming interfaces (APIs) for developing actors and edges in signal processing dataflow graphs. The APIs are not language-specific; instead, they are defined in terms of fundamental dataflow principles. In LDSS, we employ LIDE-C, which is based on C-language implementations of the LIDE APIs, and $\tau$ LIDE, which extends LIDE-C with capabilities for timed simulation [17].

\section{Modeling AND Simulation OF SNNS IN LDSS}

In LDSS, each dataflow actor corresponds to an individual neuron, and encapsulates the computations that are used to simulate the behavior of the neuron. The actor models of neurons are based on Izhikevich's model, as implied in Section III-A. Dataflow edges in LDSS correspond to synaptic connections between pairs of neurons. Given an edge $e$ in an LDSS dataflow graph, the source and sink vertices of $e$ correspond to the pre-synaptic and post-synaptic neurons associated with the connection modeled by $e$.

LDSS can be viewed as a package of SNN-specific actors and utilities that we have developed for simulating and experimenting with SNNs in $\tau$ LIDE. The utilities in LDSS include functions for automatically generating SNN models of arbitrary size using randomization techniques, and for reading SNN models from input files. This latter feature is useful to experiment with models that are generated by other SNN experimentation tools. LDSS can readily be extended with libraries that support other models for spiking neurons (beyond [10])

The dataflow graph of an SNN model in LDSS generally consists of actors that represent both excitatory neurons and inhibitory neurons. Excitatory and inhibitory neurons in LDSS are modeled as instances of two different actor types. In the SNN models studied in our experiments, the ratio of excitatory neurons to inhibitory neurons is $4: 1$.

Fig. 1 illustrates an example of a simple dataflow graph representation of an SNN in LDSS. This example involves a network of five neurons, with 4 of them $\left(E_{1}, E_{2}, E_{3}, E_{4}\right)$ being excitatory neurons and one of them $\left(I_{1}\right)$ being an inhibitory neuron. The edges in Fig. 1 model synaptic connections between neurons. In particular, a dataflow edge $(X, Y)$ in LDSS represents a synaptic connection between $\mu(X)$ and $\mu(Y)$, where $\mu(X)$ is the pre-synaptic neuron and $\mu(Y)$ is the post-synaptic neuron. Here, for a given LDSS actor $X$, we use $\mu(X)$ to denote the neuron that is represented by $X$. The numbers next to the edges shown in Fig. 1 show the weights of the corresponding synaptic connections (see Section III-A).

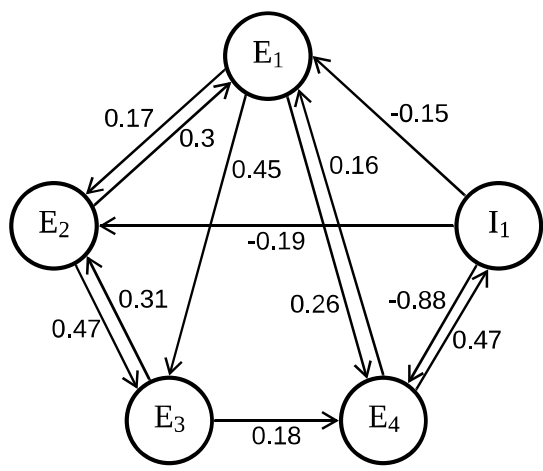

Fig. 1: An example of an SNN dataflow graph in LDSS.

Suppose that $G$ is a dataflow graph that models an SNN in LDSS, and that $N$ is an actor in $G$. The actor $N$ has an associated execution time estimation function, which is denoted by $\theta_{N}$, and computes the predicted time of the next spike that is to be generated by $\mu(N)$ based on the current state of $\mu(N)$. If no spiking event is predicted based on the current neuron state, then $\theta_{N}$ returns $\infty$. This estimation function 
performs a finite element simulation based on Izhikevich's model whenever the actor $N$ executes. The execution of $N$ in turn is driven by the arrival of spikes at the inputs of $N$ that have been generated from predecessor neurons. More specifically, the arrival of a spike at time $t$ at any input of $N$ initiates an execution of $\theta_{N}$, which in turn may produce a predicted spiking time $p_{N}(t)>t$. The magnitude of current associated with a spike is represented as a dataflow token. In this context, the term "token" is the generic term for a quantum of information that is communicated across a dataflow edge.

Each synapse is modeled with a fixed propagation delay $\delta$, which is the same for all synapses. Thus, if a neuron $\mu(A)$ generates a spike on dataflow edge $e$ at time $t_{1}$, then the spike will arrive at the input of $B$ at time $t_{2}=t_{1}+\delta$, where $B$ is the sink actor of $e$. LDSS can readily be extended with more complex propagation delay models, which represents a useful direction for future work.

At any given value $t_{a}$ of simulated time, a neuron $\mu(N)$ can have at most one predicted future spike that is pending to be processed by LDSS at some time $t_{b}>t_{a}$, where $t_{b}$ has been determined by $\theta_{N}$. Such a predicted spike is maintained as a pending event $Y_{b}$ within the event list of LDSS. However, if $\mu(N)$ already has such a predicted future spike that is pending, and a new spike arrives on an input of $N$ at some time $t_{c}$ within the interval $\left[t_{a}, t_{b}\right)$, then the previously scheduled spiking event is de-scheduled (removed from the simulator event list), and (following the rules described above for initiating an execution of $N), \theta_{N}$ is invoked to determine whether or not a new spiking event is predicted, and to compute a new predicted spiking time $p_{N}\left(t_{c}\right)$ if such an event is predicted. If the invocation of $\theta_{N}$ results in a new predicted spiking time, then the new spiking event is scheduled to occur at $p_{N}\left(t_{c}\right)$.

More specifically, if the incoming spike is from an excitatory neuron, then a new spiking event will be predicted, and based on properties of the underlying spiking neuron model, we will have $p_{N}\left(t_{c}\right)<t_{b}$. On the other hand, if the incoming spike is from an inhibitory neuron, then a new spike may or may not be predicted, depending on whether the neuron's membrane potential is lowered below $v_{\text {peak }}$. If a new spike is predicted, then the predicted time will satisfy $p_{N}\left(t_{c}\right)>t_{b}$.

Neuron $\mu(N)$ generates a spike when the membrane potential $v_{\mu(N)}$ reaches its peak value $v_{\text {peak }}$, as defined by Izhikevich's model [10]. The timing of this physical behavior is what is modeled by $\theta_{N}$. When $\mu(N)$ generates a spike at some time $t$, the actor $N$ produces a token that represents the spike on all of the $m$ output edges of $N$ in the dataflow graph.

\section{EXPERIMENTS}

We evaluated LDSS by using it to model and simulate randomly-generated SNNs with varying numbers of neurons $Q$. Specifically, we experimented with 7 randomly-generated SNNs $\Gamma_{1}, \Gamma_{2}, \ldots, \Gamma_{7}$ containing $Q=1,000,5,000,10,000$, $15,000,20,000,25,000,30,000$ neurons, respectively. For each of these SNNs, $80 \%$ of the neurons were randomly selected to be excitatory neurons, and the remaining $20 \%$ were inhibitory.

\section{A. Random SNN Generation}

We applied a random SNN generation approach proposed by Izhikevich [10]. In this approach, the edges and edge weights are derived from a randomly-generated $Q \times Q$ matrix $W$ of floating point values. Each neuron $\mu$ has a unique index $I(\mu) \in\{1,2, \ldots, Q\}$, which is used to index the rows and columns of $W$. Each network $\Gamma_{i}$ is simulated 3 times using the baseline simulator (see Section V-B), and simulated 3 times using LDSS with the exact same $\Gamma_{i}$.

The diagonal elements of $W$ are all set to 0 . Each offdiagonal element $W_{i, j}(i \neq j)$ is derived from a uniform distribution within a finite interval $\left[w_{1}, w_{2}\right]$. The endpoints of this interval differ depending on whether $n_{i}$ is excitatory or inhibitory, where $n_{i}$ represents the neuron associated with neuron index $i$ (that is, $i=I\left(n_{i}\right)$ ). If $n_{i}$ is inhibitory, then we use $w_{1}=-1, w_{2}=0$, otherwise, we use $w_{1}=0, w_{2}=0.5$. For each ordered pair $\left(n_{i}, n_{j}\right)$ of distinct neurons, we interpret $n_{i}$ to be a predecessor of $n_{j}$ if $\operatorname{abs}\left(W_{i, j}\right) \geq 0.1$, where abs represents the absolute value function. Furthermore, if $n_{i}$ is a predecessor of $n_{j}$, then the weight of the corresponding synapse is set to $W_{i, j}$.

\section{B. Baseline Simulator}

As mentioned in Section III, we evaluate LDSS by comparing its simulation output and execution time performance with a sequential, C-language implementation of SNN simulation based on Izhikevich's model [10], which is the same model used for LDSS, as described in Section IV. We refer to this sequential simulator implementation in the remainder of this paper as the baseline simulator. As its name implies, we developed the baseline simulator to provide a reference point — using the same implementation language and spiking neuron model - for evaluating the dataflow-based and eventdriven SNN simulation methods presented in this paper.

The baseline simulator uses a conventional, time-driven simulator design. It does not employ dataflow concepts or event-driven simulation techniques, which are key features of LDSS. Our simulation experiments were designed carefully to use the same underlying neuron and synapse models so that the dataflow- and event-driven simulation approach of LDSS could be functionally validated, and its performance effects isolated. This experimental approach supports the primary aim of this paper: to demonstrate the utility of the proposed new approach to integrating dataflow modeling and event-driven simulation for SNNs.

\section{Functional Validation}

To test the validity of simulation results produced by LDSS, we simulate each of the 7 randomly-generated, $Q$-neuron SNNs $\Gamma_{1}, \Gamma_{2}, \ldots, \Gamma_{7}$ using both the baseline simulator and LDSS. We simulate each network for 1,000 milliseconds (ms) of simulated time. The noise component of each neuron's background current is simulated as thalamic input, similarly as in [10]. The noise component is randomly updated at each simulated time step in the baseline simulator. In LDSS, the noise component values can be generated randomly or 


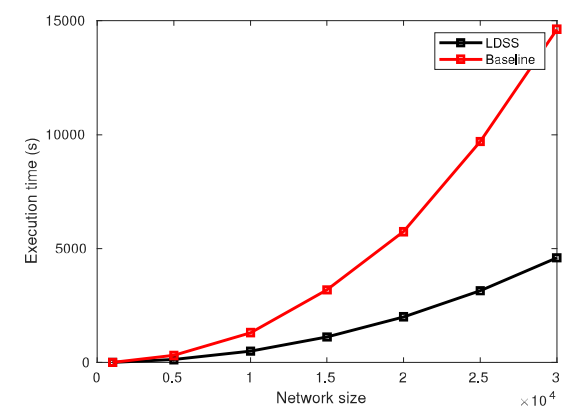

Fig. 2: Execution time for variable network size $Q$.

imported from a file. The latter option is useful when comparing output results with another simulator. For functional validation of LDSS, we import the noise components for the background currents of all neurons from a file that is exported from the baseline simulator to contain all of the randomlygenerated noise component values. This allows both simulators to operate with exactly the same noise signals. For excitatory neurons, the background current noise component is uniformly distributed in the interval $[0,5]$ pico-Amperes $(\mathrm{pA})$, and for inhibitory neurons, it is uniformly distributed in $[0,4] \mathrm{pA}$.

We compare the simulated results in terms of the average spiking frequency over all of the neurons in a given network. In each comparison, we use the same network $\Gamma_{i}$ for both the baseline simulator and LDSS. The results of these experiments show no discrepancy between the two simulators: for $\Gamma_{1}, \Gamma_{2}, \ldots, \Gamma_{n}$, both simulators report the same average spiking frequencies - $\left(4.91 \times 10^{-3}, 5.22 \times\right.$ $\left.10^{-2}, 0.12,0.14,0.17,0.21,0.22\right)$, respectively. The units of the spiking frequency values reported here are $\mathrm{kHz}-$ e.g., a value of 0.12 corresponds to $120 \mathrm{~Hz}$.

\section{Performance Comparison}

We also compared the execution time between LDSS and the baseline simulator, again using the same set of 7 randomlygenerated SNNs. All simulations were executed on the same computer, which was equipped with an Intel Core i5-8259U CPU and 16GB of RAM. The simulations were each carried out through 1 second of simulated time. The execution time results are shown in Fig. 2. The horizontal axis corresponds to the network size $Q$, and the vertical axis corresponds to the measured execution time in seconds. The results illustrate that LDSS consistently outperforms the baseline simulator, with the amount of speedup increasing with the network size.

Detailed statistics associated with the results in Fig. 2 are shown in Table I. Each row corresponds to one of the two simulators (Baseline or LDSS) applied to an SNN model with a given network size $(Q)$. The values for the mean, minimum, maximum and standard deviation (S.D.) are all reported in seconds. The speed improvement over the 7 test networks ranges from $44.45 \%$ to $68.59 \%$ with LDSS performing increasingly better compared to the baseline as $Q$ increases.
TABLE I: Execution time comparison between the baseline simulator and LDSS for different network sizes.

\begin{tabular}{|l|l|l|l|l|}
\hline Simulator, Netw. Size & Mean & Minimum & Maximum & S.D. \\
\hline Baseline, $Q=1,000$ & 6.13 & 6.06 & 6.27 & 0.12 \\
\hline LDSS, $Q=1,000$ & 3.41 & 3.27 & 3.48 & 0.12 \\
\hline Baseline, $Q=5,000$ & 310.04 & 302.86 & 317.09 & 7.11 \\
\hline LDSS, $Q=5,000$ & 127.22 & 126.98 & 127.46 & 0.24 \\
\hline Baseline, $Q=10,000$ & $1,313.36$ & $1,285.01$ & $1,335.58$ & 25.84 \\
\hline LDSS, $Q=10,000$ & 502.00 & 494.55 & 507.02 & 6.58 \\
\hline Baseline, $Q=15,000$ & $3,190.28$ & $3,184.49$ & $3,193.27$ & 5.02 \\
\hline LDSS, $Q=15,000$ & $1,125.43$ & $1,109.01$ & $1,133.65$ & 14.22 \\
\hline Baseline, $Q=20,000$ & $5,742.75$ & $5,654.09$ & $5,889.29$ & 127.84 \\
\hline LDSS, $Q=20,000$ & $2,008.45$ & $1,977.66$ & $2,023.89$ & 26.67 \\
\hline Baseline, $Q=25,000$ & $9,703.44$ & $9,617.40$ & $9,752.64$ & 74.77 \\
\hline LDSS, $Q=25,000$ & $3,149.80$ & $3,104.47$ & $3,173.70$ & 39.27 \\
\hline Baseline, $Q=30,000$ & $14,628.38$ & $14,505.39$ & $14,721.99$ & 111.24 \\
\hline LDSS, $Q=30,000$ & $4,594.30$ & $4,528.15$ & $4,631.99$ & 57.47 \\
\hline
\end{tabular}

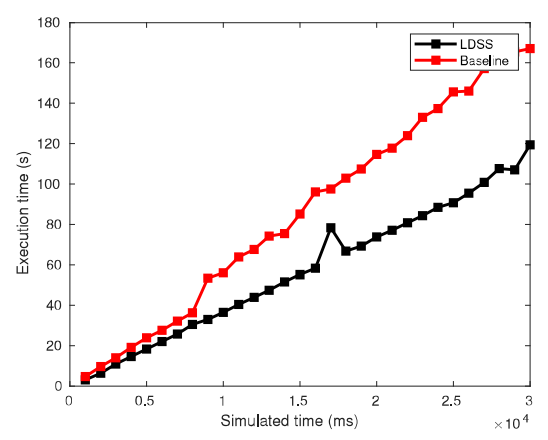

Fig. 3: Performance comparison for different amounts of simulated time.

We also compared the execution time between the baseline simulator and LDSS for a fixed network size and different amounts of simulated time. For this experiment, the network size was fixed at $Q=1,000$, and the simulated time was varied across the sequence $T=(1,000,2,000, \ldots, 30,000)$, with units in milliseconds (ms). The resulting execution time comparison is shown in Fig. 3. Each experiment is repeated 3 times, and the reported result is the average execution time across the 3 repetitions.

The results from Fig. 3 show that the relationship between execution time and simulated time is close to linear for both the baseline and LDSS simulators, with LDSS outperforming the baseline simulator by a minimum of $16.16 \%$ and maximum of $39.32 \%$ in terms of execution time.

\section{CONCLUSION}

In this paper, we have presented a dataflow-based, eventdriven approach for modeling and simulating spiking neural networks (SNNs), and we have prototyped this approach by developing a new simulation tool called the Lightweight Dataflow SNN Simulator (LDSS). Useful features of the proposed new simulation approach include improved simulation speed, enabled by the event-driven operation; design flexibility in that the simulation is not limited to a specific spiking neuron 
model or network topology; and retargetability across different hardware platforms and implementation languages, enabled by the rigorous use of dataflow modeling concepts. Interesting directions for future work include developing multi-threaded or GPU-accelerated versions of LDSS for further simulation speedup, and adaptation of other SNN simulators using models and methods from the proposed simulation approach.

\section{REFERENCES}

[1] E. Painkras et al., "SpiNNaker: A 1-W 18-core system-on-chip for massively-parallel neural network simulation," IEEE Journal of Solid State Circuits, vol. 48, no. 8, pp. 1943-1953, 2013.

[2] F. Akopyan et al., "TrueNorth: Design and tool flow of a $65 \mathrm{~mW}$ million neuron programmable neurosynaptic chip," IEEE Transactions on Computer-Aided Design of Integrated Circuits and Systems, vol. 34, no. 10, pp. 1537-1557, 2015

[3] C. Shen et al., "A lightweight dataflow approach for design and implementation of SDR systems," in Proceedings of the Wireless Innovation Conference and Product Exposition, 2010, pp. 640-645.

[4] M. L. Hines and N. T. Carnevale, "NEURON: A tool for neuroscientists," 2001.

[5] J. M. Eppler, "PyNEST: A convenient interface to the NEST simulator," Frontiers in Neuroinformatics, 2008.

[6] D. Goodman and R. Brette, "Brian: a simulator for spiking neural networks in Python," Frontiers in Neuroinformatics, vol. 2, no. 5, pp $1-10,2008$.

[7] M. Stimberg, D. F. M. Goodman, V. Benichoux, and R. Brette, "Equation-oriented specification of neural models for simulations," Frontiers in Neuroinformatics, 2014.

[8] F. Naveros, J. A. Garrido, R. R. Carrillo, E. Ros, and N. R. Luque "Event- and time-driven techniques using parallel CPU-GPU coprocessing for spiking neural networks," Frontiers in Neuroinformatics, vol. 11,2017 , article 7 .

[9] K. Cheung, S. R. Schultz, and W. Luk, "NeuroFlow: A general purpose spiking neural network simulation platform using customizable processors," Frontiers in Neuroscience, vol. 9, no. JAN, 2016.

[10] E. M. Izhikevich, "Simple model of spiking neurons," IEEE Transactions on Neural Networks, vol. 14, no. 6, pp. 1569-1572, 2003.

[11] R. Brette and W. Gerstner, "Adaptive exponential integrate-and-fire model as an effective description of neuronal activity." Journal of neurophysiology, vol. 94, pp. 3637-3642, 2005.

[12] C. Koch and I. Segev, Methods in Neuronal Modeling: From Ions to Networks, 1998.

[13] G. Bi and M. Poo, "Synaptic modifications in cultured hippocampal neurons: Dependence on spike timing, synaptic strength, and postsynaptic cell type," Journal of Neuroscience, vol. 18, no. 24, pp. 10464-10472, 1998.

[14] L. Li, P. Deaville, A. Sapio, L. Anttila, M. E. Valkama, M. Wolf, and S. Bhattacharyya, "A framework for design and implementation of adaptive digital predistortion systems," in Proceedings of the IEEE International Conference on Artificial Intelligence Circuits and Systems, Hsinchu, Taiwan, March 2019, pp. 112-116.

[15] E. A. Lee and T. M. Parks, "Dataflow process networks," Proceedings of the IEEE, pp. 773-799, May 1995.

[16] S. S. Bhattacharyya, E. Deprettere, R. Leupers, and J. Takala, Eds. Handbook of Signal Processing Systems, 3rd ed. Springer, 2019.

[17] J. Geng, H. Li, Y. Liu, Y. Liu, M. Kashef, R. Candell, and S. S. Bhattacharyya, "Model-based cosimulation for industrial wireless networks," in Proceedings of the IEEE International Workshop on Factory Communication Systems, 2018, pp. 1-10. 